\title{
Evaluation of solid breast lesions with power Doppler: value of penetrating vessels as a predictor of malignancy
}

Rositaa $\underline{\text { Ibrahim }}^{1}$, MBBS, MRad, Kartini Rahmat ${ }^{1}$, MBBS, FRCR, Farhana $\underline{\text { Fadzli }}^{1}$, MBChB, FRCR, Faizatul Izza $\underline{\text { Rozalli }}^{1}$, BMBS, FRCR,

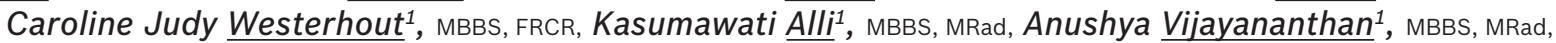

Fatimah Moosa $^{1}$, MBBS, FRCR

\begin{abstract}
INTRODUCTION This study aimed to evaluate the vascular pattern of solid breast lesions using power Doppler ultrasonography (PDUS) and assess whether the presence of intratumoural penetrating vessels can predict breast cancer malignancy.

METHODS Greyscale ultrasonography (US) and PDUS were prospectively performed on 91 women in Malaysia with histopathologically proven breast lesions. The diagnostic accuracy of greyscale US, PDUS, and both greyscale US and PDUS was calculated and compared.

RESULTS The 91 women had 102 breast lesions (55 benign, 47 malignant). Of the 47 malignant lesions, 36 demonstrated intratumoural penetrating vessels. The sensitivity, specificity, positive predictive value (PPV) and negative predictive value (NPV) of greyscale US findings in diagnosing malignancy were $100.0 \%, 71.4 \%, 74.1 \%$ and $100.0 \%$, respectively. The presence of calcification in the breast lesion and the margin, shape and posterior acoustic features of the lesion were significant parameters in predicting malignancy $(p<0.01)$. The sensitivity, specificity, PPV and NPV of the presence of intratumoural penetrating vessels in predicting malignancy were $76.5 \%, 80.0 \%, 76.5 \%$ and $80.0 \%$, respectively. When both greyscale US and PDUS were used, there was a significant correlation in predicting malignancy $(p<0.05)$. The specificity and PPV values of the combined greyscale US and PDUS method $(89.0 \%$ and $85.7 \%$, respectively) were higher than those of greyscale US or PDUS alone.

CONCLUSION Flow patterns revealed by PDUS can be useful for differentiating benign and malignant breast lesions. The visualisation of penetrating vessels in solid breast lesions can be used to complement greyscale US findings in predicting malignancy.
\end{abstract}

Keywords: breast cancer, penetrating vessel, power Doppler, ultrasonography

\section{INTRODUCTION}

According to the American Cancer Society, about 1.3 million women around the world are diagnosed with breast cancer every year and 465,000 will die from the disease. ${ }^{(1)}$ Breast cancer is the most commonly diagnosed cancer among Malaysian women. ${ }^{(2)}$ Data from the 2004 Malaysian National Cancer Registry revealed that approximately one in 20 women in Malaysia develop breast cancer during their lifetime. ${ }^{(3)}$ Although mammography is recognised as the best screening method for breast cancer, combining mammography and ultrasonography (US) helps to improve detection, especially if the woman has dense breasts. Furthermore, the detected breast lesions can be characterised more accurately when these modalities are used in combination than on their own.

The association between angiogenesis (i.e. the formation of new blood vessels) and cancer was first described by Folkman in 1971.(4) Metabolically active cancer cells demand more oxygen and nutrients than normal vessels are able to provide, resulting in hypoxia. This hypoxic state stimulates the formation of new vessels that run from existing blood vessels into the tumour in order to maintain the tumour's metabolic homeostasis. ${ }^{(5)}$
Previous studies have shown the role and significance of Doppler sonography in the evaluation of breast lesions, particularly in identifying malignancy and characterising tumours. ${ }^{(6,7)}$ Notably, the identification of tumour vascularity has been used to differentiate benign masses from malignant masses.

In the present study, we aimed to investigate the vascular pattern of solid breast lesions and determine whether the presence of penetrating vessels on power Doppler sonograms can be used to distinguish malignant lesions from benign lesions. We used power Doppler US (PDUS) instead of colour Doppler, as the former is not angle-dependent and has increased sensitivity to flow (i.e. it can detect flow in small vessels). In addition, in contrast to colour Doppler, problems related to aliasing do not occur with PDUS. ${ }^{(8,9)}$

\section{METHODS}

This study was performed in a breast imaging unit of University Malaya Medical Centre, Kuala Lumpur, Malaysia, over a period of 24 months (December 2008-December 2010). Women who presented to the unit for mammography and/or breast US, for screening and/or diagnostic indications, were eligible for inclusion. We prospectively recruited 91 consecutive female patients who 
were scheduled for US-guided tissue core biopsy, hook wire excisional biopsy or mastectomy. Both conventional US and Doppler examinations were conducted for these women. Patients who had undergone surgery or neoadjuvant chemotherapy for the lesion that was being assessed were excluded. Informed consent for examination and biopsy was obtained from the participants. Approval for this study was obtained from the University of Malaya Research Ethics Committee.

The final histopathological diagnoses were confirmed using the results of tissue core biopsy or excisional biopsy, or the mastectomy specimen, when available. Diagnostic B-mode greyscale US and PDUS imaging were performed using a medical grade US system (Philips iU22; Philips Healthcare, Bothell, WA, USA) with a high frequency (i.e. $12.5 \mathrm{MHz}$ ) linear probe. Two radiologists performed breast US after the examination protocol had been standardised via a one-week trial run involving ten patients. The images were captured as static and cine images on the scanner, and saved in blinded datasets for independent interpretation and review. Each breast lesion was evaluated using (a) conventional B-mode greyscale US alone; and (b) a combination of B-mode greyscale US and PDUS in both the supine and supine oblique positions. B-mode greyscale US was done in the transverse and perpendicular planes to allow assessment of the lesion's size and morphologic characteristics. For each patient, one radiologist performed both the B-mode greyscale US and PDUS, while another radiologist reviewed and analysed the imaging dataset at a separate setting. The PDUS gain was maintained between $70 \%$ and $80 \%$, and minimal pressure was applied to the skin to avoid vascular compression by the US probe.

The morphology of the breast lesions on B-mode greyscale US was characterised according to the American College of Radiology's Breast Imaging-Reporting and Data System (ACR BI-RADS ()$_{)}{ }^{(10)}$ The size, shape, echogenicity, orientation, margin and posterior acoustic features of the lesion, as well as the presence of calcification in the lesion, were documented. Lesions with features that were typically benign and had no suspicious findings were categorised as benign or probably benign (i.e. BI-RADS 2 or 3). In our institution, patients with lesions that are categorised as probably benign (i.e. BI-RADS 3) may undergo percutaneous biopsy if they have an increased risk of breast cancer, previous surgery for high-risk lesions, or large lesions scheduled for excision surgery. Features were documented if they were suspicious of or indicated malignancy, such as irregular shape, non-circumscribed margins (e.g. spiculated or microlobulated margins), microcalcifications, taller-than-wide (non-parallel) orientation, heterogeneous internal echogenicity and ductal extension. If there was only one suspicious finding, the lesion would be categorised as BI-RADS 4 (i.e. suspicious for malignancy). If there were two or more suspicious findings, the lesion would be categorised as BI-RADS 5 (i.e. highly suspicious for malignancy).

The vascular patterns of the lesions on PDUS were categorised according to the groups described by Raza and Baum: ${ }^{(9)}$ (a) no vessels - no flow was observed within the lesion; (b) peripheral or central vessels - vessels were observed within the lesion or curved along the margin of the lesion (Fig. 1); (c) penetrating vessels - vessels were observed arising from the edge of the lesion

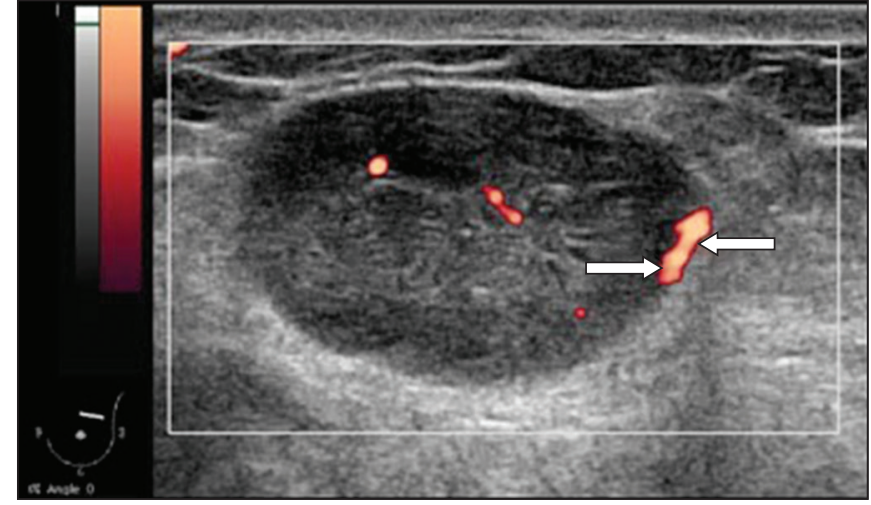

Fig. 1 Power Doppler US image shows vascular flow at the centre and along the periphery of the mass (arrows).

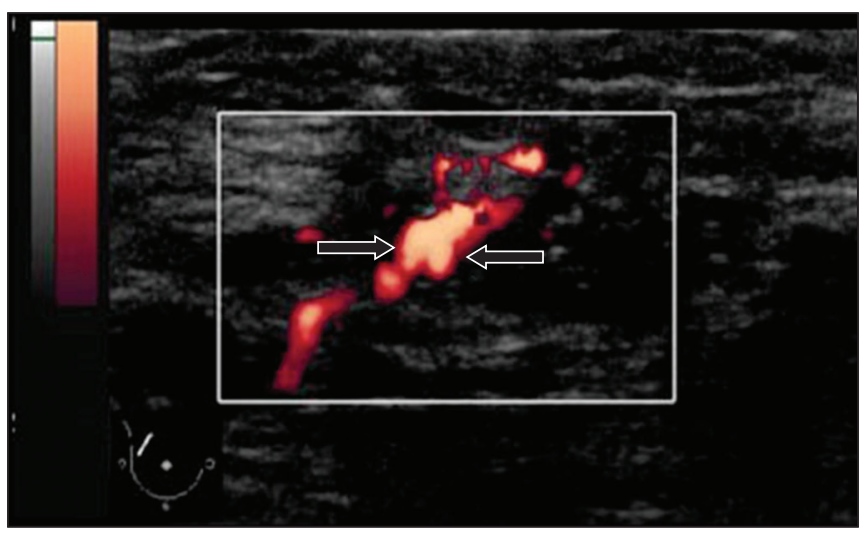

Fig. 2 Power Doppler US image shows penetrating vessels in an irregular hypoechoic mass. The vessels are seen coursing into the mass (arrows).

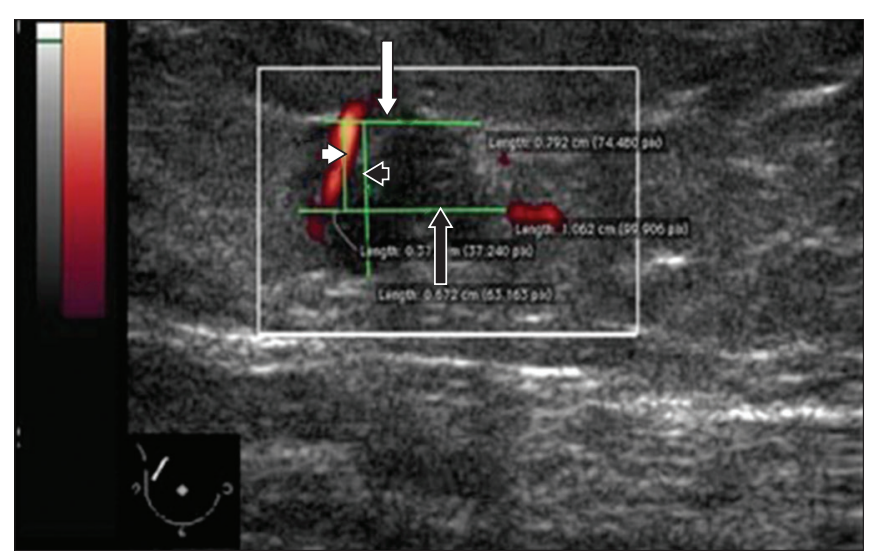

Fig. 3 Power Doppler US image shows how the variables, Depth 1 and Depth 2, are measured to calculate the degree of vascular penetration. A line is drawn at the superficial margin of the lesion (white arrow), perpendicular to the point of entry of the penetrating vessel. Another line is then drawn, parallel to the previous line at the point where the penetrating vessel ends (black arrow). Depth 1 (white arrowhead), which is the depth/length of the penetrating vessel in the lesion, is the distance between the two parallel lines. Depth 2 (black arrowhead) is the depth of the lesion.

and traversing into the lesion, with or without a branching pattern (Fig. 2). The degree of vascular penetration (in percentage) was quantitatively calculated using the following formula (Fig. 3):

\section{$\frac{\text { Depth } 1}{\text { Depth } 2} \times 100$}

The US image datasets were reviewed and interpreted by two radiologists who specialise in breast imaging. They were blinded 


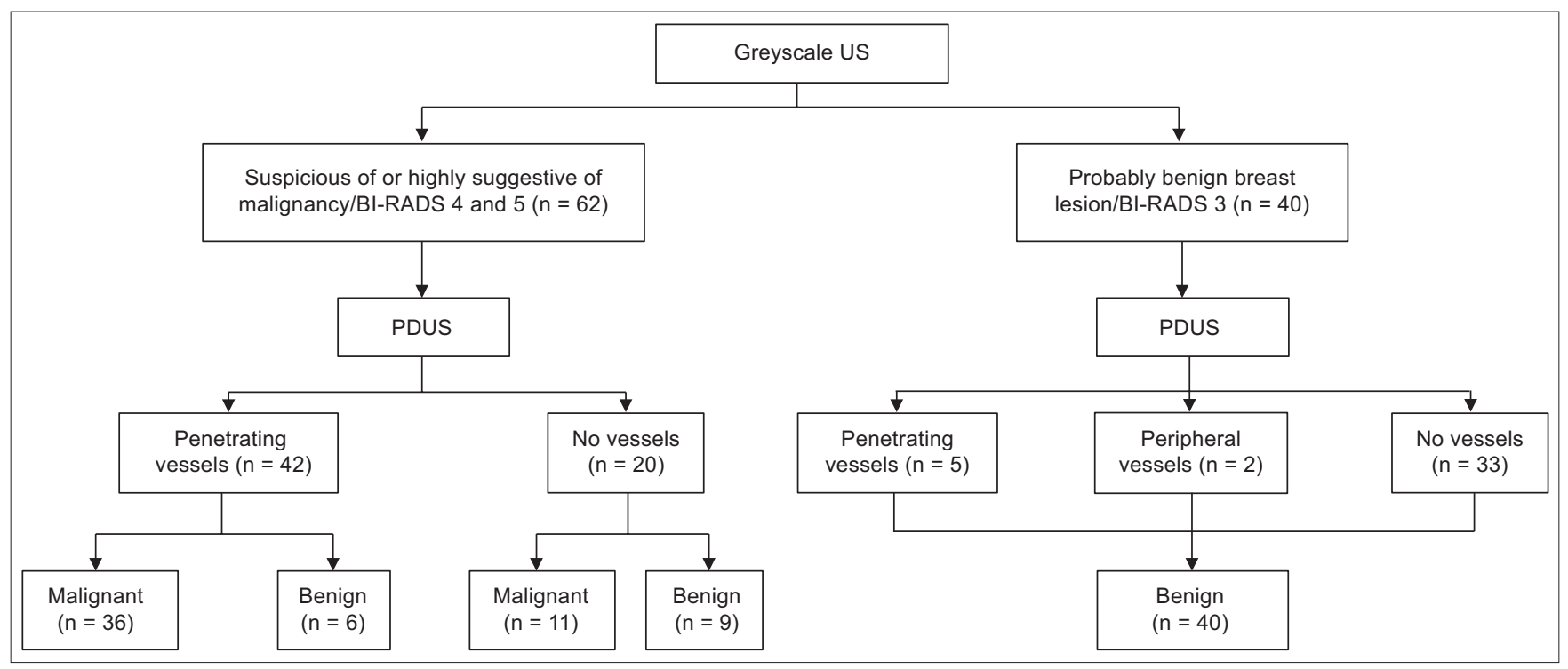

Fig. 4 Flow chart shows the classification of the patients' breast lesions when both B-mode greyscale ultrasonography (US) and power Doppler US (PDUS) were used $(n=102)$.

to the patients' clinical information, mammography findings and final histopathology diagnosis. Each breast lesion was assigned an appropriate BI-RADS category based on its morphological appearance. The vascular patterns of the lesions were categorised according to the groups described in the previous paragraph, once by using the PDUS images alone, and a second time by using the PDUS images in combination with the B-mode greyscale US images. The histopathological diagnosis was used as the reference standard, and the definite result from the mastectomy specimen, excisional biopsy or tissue core biopsy was used for correlation and statistical analysis.

The data was tabulated into a Microsoft Excel spreadsheet (Microsoft, Redmond, WA, USA) and statistical analysis was performed using SPSS Statistics version 17.0 (SPSS Inc, Chicago, USA). Pearson's chi-square test and Mann-Whitney $U$ test were used to test for correlations between variables. The sensitivity, specificity, positive predictive value (PPV) and negative predictive value (NPV) were calculated for the vascular pattern and depth ratio.

\section{RESULTS}

A total of 91 women, aged 18-68 years, were included in the study. Collectively, there were 102 breast lesions among the women: 47 were malignant and 55 were benign. Among the malignant lesions, 45 were invasive ductal carcinomas (IDCs), one was a mucinous carcinoma and one was a ductal carcinoma in situ. Among the benign lesions, 31 were fibroadenomas, 15 were benign proliferative lesions, two were due to fibrocystic disease, two were benign phyllodes tumours, two were due to granulomatous mastitis, one was a fibrofatty lesion, one was atypical ductal hyperplasia and one was an intraductal papilloma. Patients with malignant breast lesions underwent either breast-conserving surgery or mastectomy, except for two patients with bilateral advanced carcinoma who underwent chemotherapy and radiotherapy.

In our study cohort, breast carcinoma was most common among the Chinese $(n=20)$, followed by Malays $(n=15)$ and
Indians ( $\mathrm{n}=8$ ). The higher incidence of breast carcinoma among Chinese women corresponds with the data from the Malaysian Cancer Registry 2004. ${ }^{(3)}$ However, unlike the 2004 data, the incidence of breast carcinoma in the present study was higher among Malay patients compared to Indian patients. ${ }^{(3)}$ The mean age of the women with malignant lesions was 52 (range 24-68) years, while the mean age of the women with benign lesions was 36 (range 18-66) years. The size of the benign lesions ranged from 3-98 mm, while the size of the malignant lesions ranged from 7-53 $\mathrm{mm}$. Using only the B-mode greyscale US images, the 102 breast lesions were prospectively categorised as either: (a) probably benign/BI-RADS $3(\mathrm{n}=40)$; or (b) suspicious or highly suggestive of malignancy/BI-RADS 4 or $5(n=62)$. All the BI-RADS 3 breast lesions were revealed to be benign on histopathology. Among the 62 lesions that were categorised as suspicious or highly suspicious of malignancy/BI-RADS 4 or 5 , 47 were malignant on histopathology. Fig. 4 summarises the results of the classification when both B-mode greyscale US and PDUS were used.

The sensitivity, specificity, PPV and NPV of B-mode greyscale US alone in predicting malignancy were $100.0 \%$, $71.4 \%, 74.1 \%$ and $100.0 \%$, respectively (Table I). The margin, shape, posterior acoustic features and presence of calcification were the significant parameters $(p<0.01)$ for differentiating histologically malignant lesions from histologically benign lesions (Table II). Non-circumscribed margin, irregular shape, posterior shadowing and calcification were features that had a high PPV for malignancy (PPV 74.6\%, 87.5\%, 85.0\% and 88.9\%, respectively). In the present study, the taller-than-wide feature did not show a significant association with the probability of malignancy. While most of the benign lesions showed well-circumscribed margins, a regular shape, the absence of shadowing or posterior enhancement, and no calcifications, 12 benign lesions had an irregular shape, 15 had non-circumscribed margins and three had posterior shadowing. 
Table I. Comparison of the diagnostic accuracy of breast lesion assessment using B-mode greyscale ultrasonography (US), power Doppler US, and combined B-mode greyscale US and power Doppler US.

\begin{tabular}{|c|c|c|c|c|}
\hline \multirow[t]{2}{*}{ Variable } & \multicolumn{4}{|c|}{$\%$} \\
\hline & Sensitivity & Specificity & PPV & NPV \\
\hline \multicolumn{5}{|l|}{ B-mode greyscale US } \\
\hline BI-RADS 4/5 vs. BI-RADS 3 & 100.0 & 71.4 & 74.1 & 100.0 \\
\hline \multicolumn{5}{|l|}{ Power Doppler US } \\
\hline Penetrating vessel vs. no central/peripheral vessel & 76.5 & 80.0 & 76.5 & 80.0 \\
\hline $\begin{array}{l}\text { BI-RADS } 4 / 5 \text { and penetrating vessel vs. BI-RADS } 3 \\
\text { and no central/peripheral vessel }\end{array}$ & 76.5 & 89.0 & 85.7 & 81.0 \\
\hline
\end{tabular}

BI-RADS: Breast Imaging-Reporting and Data System; NPV: negative predictive value; PPV: positive predictive value

Table II. Summary of the B-mode greyscale ultrasonography (US) and power Doppler US (PDUS) features of the 102 breast lesions.

\begin{tabular}{|c|c|c|c|}
\hline \multirow[t]{2}{*}{ Feature } & \multicolumn{2}{|c|}{ No. (\%) } & \multirow[t]{2}{*}{ p-value } \\
\hline & Benign ( $n=55)$ & Malignant ( $n=47)$ & \\
\hline \multicolumn{4}{|l|}{ Shape } \\
\hline Regular $(n=54)$ & $49(90.7)$ & $5(9.3)$ & 0.01 \\
\hline Irregular $(n=48)$ & $6(12.5)$ & $42(87.5)$ & 0.01 \\
\hline \multicolumn{4}{|l|}{ Margin } \\
\hline Non-circumscribed $(n=59)$ & $15(25.4)$ & $44(74.6)$ & 0.01 \\
\hline \multicolumn{4}{|l|}{ Orientation } \\
\hline Parallel $(n=89)$ & $50(56.2)$ & $39(43.8)$ & 0.20 \\
\hline Non-parallel $(n=13)$ & $5(38.5)$ & $8(61.5)$ & 0.40 \\
\hline \multicolumn{4}{|l|}{ Calcification } \\
\hline Not present $(n=93)$ & $54(58.1)$ & $39(41.9)$ & 0.01 \\
\hline Present $(n=9)$ & $1(11.1)$ & $8(88.9)$ & 0.01 \\
\hline \multicolumn{4}{|l|}{ Posterior acoustic features } \\
\hline None, posterior acoustic enhancement $(n=82)$ & $52(63.4)$ & $30(36.6)$ & 0.01 \\
\hline Posterior shadowing $(n=20)$ & $3(15.0)$ & $17(85.0)$ & 0.01 \\
\hline \multicolumn{4}{|l|}{ PDUS } \\
\hline No vessel/non-penetrating vessel $(n=55)$ & $44(80.0)$ & $11(20.0)$ & 0.01 \\
\hline Penetrating vessel $(n=47)$ & $11(23.4)$ & $36(76.6)$ & 0.01 \\
\hline
\end{tabular}

When the breast lesions were evaluated using PDUS, 47 lesions demonstrated penetrating vessels and two lesions had peripheral vessels. Among the 47 breast lesions with penetrating vessels, 42 were classified as BI-RADS 4 or 5, and five were classified as BI-RADS 3. Histopathological examination showed that $36(76.6 \%)$ of 47 breast lesions with penetrating vessels were malignant and $11(20.0 \%)$ were benign (six fibroadenomas, one giant fibroadenoma, one ductal hyperplasia, one papilloma, one mastitis/fat necrosis and one granulomatous mastitis). A peripheral vessel pattern was observed in two lesions that were categorised as BI-RADS 3; these two lesions were histologically proven to be fibroadenomas. There were 11 malignant lesions that did not exhibit any vascularity - nine were IDCs, one was a tubular carcinoma and one was a mucinous carcinoma. The results of the chi-square and Mann-Whitney $U$ tests showed that there was a significant correlation between the presence of penetrating vessels and malignancy $(p<0.05)$ (Table II). The sensitivity, specificity, PPV and $\mathrm{NPV}$ of the presence of penetrating vessels in predicting malignancy were $76.5 \%, 80.0 \%, 76.5 \%$ and $80.0 \%$, respectively (Table I).
When B-mode greyscale US findings were combined with PDUS findings, chi-square test results showed that the combined findings were able to differentiate benign lesions from malignant lesions ( $p<0.05$ for all features except for tumour orientation) (Table II). The sensitivity, specificity, PPV and NPV for the combined B-mode greyscale US and PDUS method were $76.5 \%$, $89.0 \%, 85.7 \%$ and $81.0 \%$, respectively (Table I).

The degree of vascular penetration ranged from $5.4 \%-100 \%$ for malignant lesions and $10.2 \%-60.1 \%$ for benign lesions. Using the Mann-Whitney $U$ test, we found that there was no correlation between the degree of vascular penetration and malignancy $(p>0.05)$. Examples of IDCs that had varying degrees of vascular penetration are shown in Figs. 5-7; linear penetrating vessels are shown in Fig. 5, while peripheral and branching vessels are shown in Fig. 7.

\section{DISCUSSION}

US is an established, adjuvant diagnostic tool to mammography for characterising breast lesions and differentiating between 

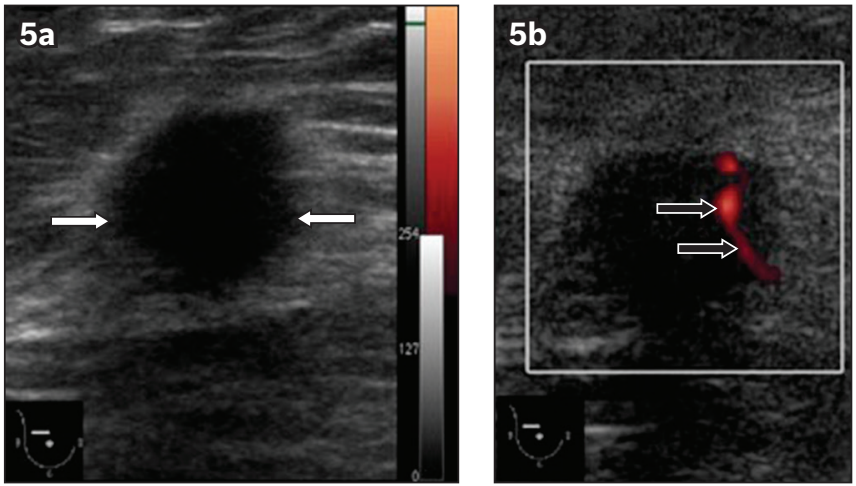

Fig. 5 An invasive ductal carcinoma in the right breast of a 51-year-old woman. (a) B-mode greyscale US image shows an irregular hypoechoic mass with spiculated margins. The mass appears to have a taller-than-wide (non-parallel) orientation with associated architectural distortion (white arrows). (b) Power Doppler US image shows linear penetrating vessels traversing the mass (black arrows).
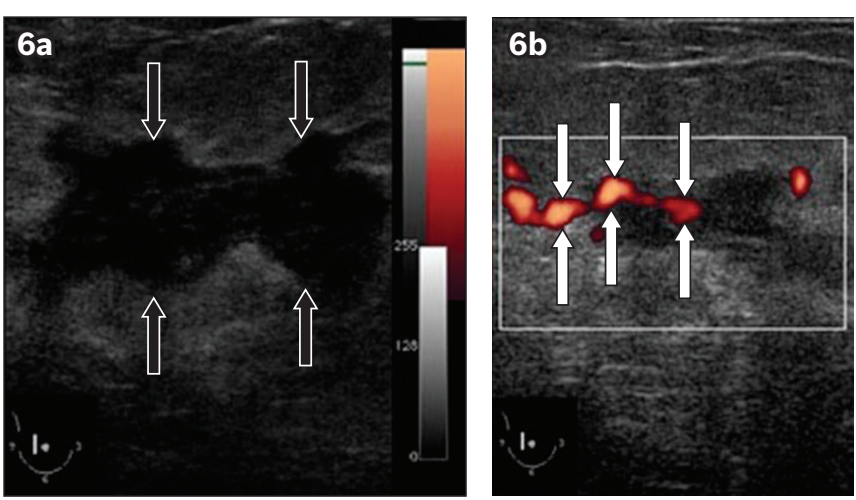

Fig. 6 An invasive ductal carcinoma in the right breast of a 55-year-old woman. (a) B-mode greyscale US image shows an irregular $3 \mathrm{~cm}$ solid hypoechoic mass with microlobulated margins (black arrows). Minimal posterior shadowing is also seen. This mass was interpreted as a highly suspicious lesion (i.e. categorised as BI-RADS 5). (b) Power Doppler US image shows vessels penetrating into the mass (white arrows).
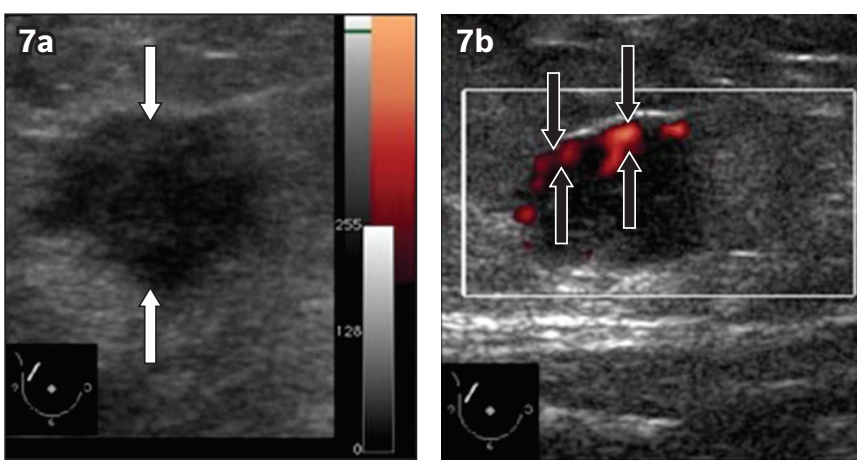

Fig. $7 \mathrm{~A}$ lesion in the right breast of a 47-year-old woman who presented with a palpable right breast mass. (a) B-mode greyscale US image shows an irregular hypoechoic lesion that has a taller-than-wide orientation (white arrows) and spiculated margins. (b) Power Doppler US image shows peripheral and branching penetrating vessels in the lesion (black arrows). The lesion was histopathologically diagnosed as an invasive ductal carcinoma.

benign and malignant lesions. The ACR BI-RADS US lexicon classification is a standard guideline that is widely used in the evaluation and categorisation of breast lesions (as benign, probably benign, suspicious and highly suspicious of malignancy). ${ }^{(10)}$ The growth of a breast cancer is closely associated with abnormal new blood vessel formation or angiogenesis. The cancer cells stimulate the development of abnormal new vessels through pre-existing microvessels within the normal breast tissue. A study by McNicholas et al showed that the presence of intratumoural vessels was significantly associated with malignancy in both palpable and non-palpable breast lesions. ${ }^{(11)}$ In fact, one study showed that the presence of intratumoural vessels can be used to determine the prognosis of breast cancer as well as the therapeutic approach for it. ${ }^{(12)}$

In the present study, the breast lesions were first evaluated using B-mode greyscale US, according to the ACR BI-RADS criteria, followed by PDUS (to assess vascular patterns). Based on the B-mode greyscale US findings, there was a significant correlation between BI-RADS categorisation and histopathology results $(p<0.05)$. The sensitivity, specificity, PPV and NPV of B-mode greyscale US were $100.0 \%, 71.4 \%, 74.1 \%$ and $100.0 \%$, respectively; the specificity and PPV were higher than those reported by Gokalp et al and Kwak et al, while the sensitivity and NPV were similar to those reported by Kwak et al, but higher than those reported by Gokalp et al. ${ }^{(13,14)}$ The improvement in specificity and PPV could be due to the use of a high-resolution US probe.

According to the ACR BI-RADS criteria, the presence of a single malignant feature in a breast lesion renders it a BI-RADS 4 or 5 lesion (i.e. suspicious or highly suspicious of malignancy). In the present study, most of the lesions that were suspicious or highly suspicious of malignancy exhibited at least two suspicious sonographic features. Our finding that the shape, margin, posterior acoustic features, and the presence of calcification can be used to predict whether the lesions were benign or malignant is comparable to those of previous studies..$^{(9,13-16)}$

In terms of breast lesion orientation, unlike the study by Gokalp et al, ${ }^{(13)}$ we did not find the taller-than-wide feature to be significantly associated with malignancy. This could be due to the small sample size of the present study or the size of the lesions examined, which were mostly $>2 \mathrm{~cm}$. In a lesion with a taller-than-wide orientation, the long axis is not parallel to the skin line. This feature has been previously shown to correlate well with malignancy. In contrast, wider-than-tall or oval-shaped lesions (i.e. long axis parallel to the skin line) are associated with benignity. ${ }^{(16)}$ In the present study, 13 of the 102 breast lesions had the taller-than-wide feature; however, only eight lesions were confirmed to be malignant on histological examination.

We selected PDUS over colour Doppler US to evaluate the vascular pattern of lesions, as the former has a higher sensitivity in detecting flow in solid breast lesions, particularly if small vessels and low-velocity blood flow are involved. ${ }^{(17)}$ Since PDUS measures the amplitude of blood flow, it is angle-independent and avoids aliasing artefacts. ${ }^{(18,19)}$ Although PDUS has shown great potential in predicting malignancy, ${ }^{(16,19,20)}$ its use is limited by the lack of standard interpretation guidelines. Hence, we aimed to assess whether the vascular pattern of lesions can be used to predict malignancy and whether the vascular pattern of a lesion correlates with its BI-RADS category. Although other aspects of PDUS imaging could have been used, evaluating vascular patterns appears to be less time-consuming compared to other parameters 
such as the resistive index. The morphology of the vessels of solid breast lesions on PDUS was first described by Raza and Baum, ${ }^{(9)}$ who found that the sensitivity, specificity, PPV and NPV of using penetrating vessels to predict malignancy were $68 \%, 95 \%, 85 \%$ and $88 \%$, respectively. The vascular patterns of the lesions, as seen on PDUS, correlated with the histopathology results in the present study, with high specificity and NPV (Table I). The degree of vascular penetration within the breast mass was also calculated, but there was no significant correlation between the degree of vascular penetration and malignancy. In other words, the depth of vessel penetration did not contribute to the characterisation of lesions in the present study. Thus, we conclude that the presence of penetrating vessels alone, regardless of the degree of penetration, is a good predictor of malignancy.

We found that the presence of penetrating vessels in combination with suspicious or highly suspicious features (i.e. BI-RADS 4 or 5) yielded the best diagnostic accuracy. The specificity and PPV were high (specificity $89.0 \%$, PPV $85.7 \%$ ) in predicting malignancy, as compared to the diagnostic accuracy of penetrating vessels alone (specificity $80.0 \%$, PPV 76.5\%) and BI-RADS features alone (specificity $71.4 \%$, PPV $74.1 \%$ ). The number of false positive results in the combined assessment group was also lower than that of the group that assessed penetrating vessels alone and the group that assessed BI-RADS features alone. Although BI-RADS categorisation is a sensitive method for predicting malignancy, it produces a greater number of false positive results (i.e. benign lesions that show malignant morphology sonographically) due to its low specificity. When the presence of penetrating vessels was used to predict malignancy in the present study, false positive results were seen in 11 benign lesions. Penetrating vessels were seen in six fibroadenomas, one giant fibroadenoma, one atypical ductal hyperplasia, one papilloma, one mastitis/fat necrosis and one granulomatous mastitis. Thus, while the malignancy pick-up rate improved with the use of PDUS, sensitivity and NPV were reduced, resulting in an increased biopsy rate; this is similar to the results of a study conducted by Tozaki and Fukuma. ${ }^{(21)}$

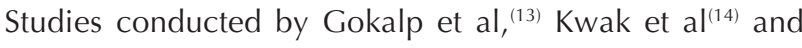
Lee et $\mathrm{al}^{(20)}$ on the role of PDUS in predicting malignancy yielded results that were similar to those of the present study. However, we obtained a higher diagnostic accuracy of PPV and specificity for the combined B-mode greyscale US and PDUS method as compared to Kwak et al. ${ }^{(14)}$ The diagnostic accuracy achieved based on vessel penetration alone was relatively similar to that reported by Gokalp et al. ${ }^{(13)}$ One of the limitations of the present study is the possibility of bias and interobserver variability during the B-mode greyscale US and PDUS image acquisition, since it was performed in the same sitting by the two radiologists. In an attempt to alleviate interobserver variability, we conducted an initial trial run to standardise image acquisition and interpretation of the US and PDUS images.

The recent years have seen promising technological advances in breast US. Several breast cancer studies showed increased diagnostic accuracy with the addition of strain and shearwave elastography to B-mode greyscale US. ${ }^{(22,23)}$ Cho et al also demonstrated increased diagnostic performance in distinguishing benign from malignant breast masses using combined method US elastography and colour Doppler US. ${ }^{(24)}$ The authors proposed that follow-up US, instead of biopsy, can be recommended to patients with breast masses that have a low index of suspicion for malignancy (i.e. avascular on colour Doppler imaging and showing no stiffness on elastography). ${ }^{(24)}$ The newer US scanners are readily equipped with manufacturer-specific elastography software, which are beneficial in the assessment of indeterminate and equivocal breast lesions.

Breast US is a useful adjunct to mammography in breast cancer detection and can be used to characterise breast lesions. The vascular flow patterns of breast lesions on PDUS may be useful for the differentiation of benign and malignant breast lesions. The present study showed an improvement in specificity for predicting malignancy when suspicious lesions were evaluated using both B-mode greyscale US and PDUS. Visualisation of penetrating vessels in solid breast lesions using PDUS can be used to complement B-mode greyscale US in the assessment of breast lesions, so as to improve the prediction of malignancy and determine when histopathological assessment is warranted.

\section{ACKNOWLEDGEMENTS}

The authors would like to thank Dr Mohammad Nazri Mohd Shah, Clinical Radiologist; Leong Sook Sam, Sonographer; and Norhana Mohd Nor, Sonographer, University Malaya Medical Centre, Kuala Lumpur, Malaysia, for their technical assistance. This study received financial assistance from the following institutional grants: RP008B-13HTM, FS359/2008C and RP008C-13HTM.

\section{REFERENCES}

1. Smith RA, Saslow D, Sawyer KA, et al; American Cancer Society High-Risk Work Group; American Cancer Society Screening Older Women Work Group; American Cancer Society Mammography Work Group; American Cancer Society Physical Examination Work Group; American Cancer Society New Technologies Work Group; American Cancer Society Breast Cancer Advisory Group. American Cancer Society guidelines for breast cancer screening: update 2003. CA Cancer J Clin 2003; 53:141-69.

2. Hisham AN, Yip CH. Spectrum of breast cancer in Malaysian women: overview. World J Surg 2003; 27:921-3.

3. Yip CH, Taib NA, Mohamed I. Epidemiology of breast cancer in Malaysia. Asian Pac J Cancer Prev 2006; 7:369-74.

4. Folkman J. Tumor angiogenesis: therapeutic implications. N Engl J Med 1971; 285:1182-6.

5. Milz P, Lienemann A, Kessler M, Reiser M. Evaluation of breast lesions by power Doppler sonography. Eur Radiol 2001; 11:547-54.

6. Cosgrove DO, Kedar RP, Bamber JC, et al. Breast diseases: color Doppler US in differential diagnosis. Radiology 1993; 189:99-104.

7. Holcombe C, Pugh N, Lyons K, et al. Blood flow in breast cancer and fibroadenoma estimated by colour Doppler ultrasonography. Br J Surg 1995; 82:787-8.

8. Moon WK, Im JG, Noh DY, Han MC. Nonpalpable breast lesions: evaluation with power Doppler US and a microbubble contrast agentinitial experience. Radiology 2000; 217:240-6.

9. Raza S, Baum JK. Solid breast lesions: evaluation with power Doppler US. Radiology 1997; 203:164-8.

10. D'Orsi CJ, Sickles EA, Mendelson EB, et al. ACR BI-RADS $®$ Atlas, Breast Imaging Reporting and Data System. Reston, VA: American College of Radiology, 2013.

11. McNicholas MM, Mercer PM, Miller JC, et al. Color Doppler sonography in the evaluation of palpable breast masses. AJR Am J Roentgenol 1993; 161:765-71

12. Stuhrmann M, Aronius R, Schietzel M. Tumor vascularity of breast lesions 
potentials and limits of contrast-enhanced Doppler sonography. AJR Am Roentgenol 2000; 175:1585-9.

13. Gokalp G, Topal U, Kizilkaya E. Power Doppler sonography: anything to add to BI-RADS US in solid breast masses? Eur J Radiol 2009; 70:77-85.

14. Kwak JY, Kim EK, Kim MJ, et al. Power Doppler sonography: evaluation of solid breast lesions and correlation with lymph node metastasis. Clin Imaging 2008; 32:167-71.

15. Hong AS, Rosen EL, Soo MS, Baker JA. BI-RADS for sonography: positive and negative predictive values of sonographic features. AJR Am Roentgenol 2005; 184:1260-5.

16. Stavros AT, Thickman D, Rapp CL, et al. Solid breast nodules: use of sonography to distinguish between benign and malignant lesions. Radiology 1995; 196:123-34.

17. Kook SH, Park HW, Lee YR, et al. Evaluation of solid breast lesions with power Doppler sonography. J Clin Ultrasound 1999; 27:231-7.

18. Mehta TS, Raza S, Baum JK. Use of Doppler ultrasound in the evaluation of breast carcinoma. Semin Ultrasound CT MR 2000; 21:297-307.

19. Piñero A, Reus M, Illana J, et al. Palpable breast lesions: utility of Doppler sonography for diagnosis of malignancy. Breast 2003; 12:258-63.

20. Lee SW, Choi HY, Baek SY, Lim SM. Role of color and power doppler imaging in differentiating between malignant and benign solid breast masses. J Clin Ultrasound 2002; 30:459-64.

21. Tozaki M, Fukuma E. Does power Doppler ultrasonography improve the BI-RADS category assessment and diagnostic accuracy of solid breast lesions? Acta Radiol 2011; 52:706-10.

22. Evans A, Whelehan $\mathrm{P}$, Thomson $\mathrm{K}$, et al. Differentiating benign from malignant solid breast masses: value of shear wave elastography according to lesion stiffness combined with greyscale ultrasound according to $\mathrm{BI}$ RADS classification. Br J Cancer 2012; 107:224-9.

23. Alhabshi SM, Rahmat K, Abdul Halim N, et al. Semi-quantitative and qualitative assessment of breast ultrasound elastography in differentiating between malignant and benign lesions. Ultrasound Med Biol 2013; 39:568-78.

24. Cho $N$, Jang $M$, Lyou CY, et al. Distinguishing benign from malignant masses at breast US: combined US elastography and color doppler US influence on radiologist accuracy. Radiology 2012; 262:80-90. 